\title{
Modulation of neural activity by angle of rotation during imagined spatial transformations
}

\author{
Madeleine Keehner ${ }^{1}$, Scott A. Guerin ${ }^{2}$, \\ Michael B. Miller², David J. Turk³, \\ Mary Hegarty ${ }^{2}$ \\ ${ }^{1}$ Curtin University of Technology \\ ${ }^{2}$ University of California, Santa Barbara \\ ${ }^{3}$ University of Aberdeen
}

$\underline{\text { Address for correspondence: }}$

Madeleine Keehner

School of Psychology

Curtin University of Technology

GPO Box U 1987, Perth 6845

Western Australia

Email: m.keehner@curtin.edu.au

Tel: (+61 8) 9266 7183; Fax: (+61 8) 92662464. 


\begin{abstract}
Imagined spatial transformations of objects (e.g., mental rotation) and the self (e.g., perspective taking) are psychologically dissociable. In mental rotation, the viewer transforms the location or orientation of an object relative to stable egocentric and environmental reference frames. In imagined shifts of perspective, the viewer's egocentric reference frame is transformed with respect to stable objects and environment. Using fMRI we showed that during mental transformations of objects the right superior parietal cortex exhibited a positive linear relationship between hemodynamic response and degrees of rotation. By contrast, during imagined transformations of the self, the same regions exhibited a negative linear trend. We interpret this finding in terms of the role of parietal cortex in coding the locations of objects in relation to the body.
\end{abstract}


To imagine an object or scene with a change in orientation, we can imagine either that the object or scene moves while we remain in place (e.g., mental rotation) or that we move to view it from a different location (perspective taking). Both mental rotation and perspective taking involve processes of encoding, maintaining, and transforming spatial information, but they require different manipulations of spatial reference frames. During mental rotation, the viewer imagines changes to an object's position with respect to his or her own egocentric or viewer-centered reference frame and the reference frame of the surrounding environment, which remain stable. During imagined shifts of perspective, by contrast, the viewer's egocentric reference frame is transformed while the reference frames of objects and environment remain stable.

Although highly correlated (Carroll, 1993), both psychometric (Hegarty \& Waller, 2004; Kozhevnikov \& Hegarty, 2001) and experimental (Huttenlocher \& Presson, 1973, 1979; Wraga, Creem, \& Proffitt, 2000) studies support a dissociation between mental rotation and perspective taking (for a recent review, see Zacks \& Michelon, 2005). For example, tasks requiring object-rotations and self-rotations sometimes produce different response time profiles. Response times (RTs) for mental rotation increase linearly with angle of rotation (Shepard \& Metzler, 1971), suggesting an analog process. By contrast RTs from imagined self-rotations do not show a consistent pattern, with some studies showing a linear increase, although typically not as great as in mental rotation (Easton \& Sholl, 1995; Reiser, 1989), and others indicating RTs that do not differ systematically with rotation angle (Creem et al., 2001; Wraga et al., 2000). While several neuroimaging studies have examined mental rotation (e.g., Carpenter, Just, Keller, \& Eddy, 1999; Cohen et al., 1996; Harris et al., 2000; 
Podzebenko, Egan, \& Watson, 2002; Richter et al., 2000), relatively few have examined the neural activation associated with perspective taking (Creem et al., 2001) or directly compared the two processes using comparable tasks (Wraga, Shepard, Church, Inati, \& Kosslyn, 2005; Zacks, Ollinger, Sheridan, \& Tversky, 2002; Zacks, Rypma, Gabrieli, Tversky, \& Glover, 1999; Zacks, Vettel, \& Michelon, 2003). A particularly informative paradigm is one in which participants see the same stimuli and make the same responses in the two tasks so that the only difference is in the spatial transformation to be executed. Thus by comparing activation in the two tasks it is possible to isolate any neural activity specific to each type of transformation, rather than processes common to both such as encoding and responding.

Using this approach, Zacks et al. (2003) found greater activity in right parietal cortex, specifically in the intraparietal sulcus (BA 7/40), during mental rotation. By contrast, imagined shifts of perspective were associated with greater modulation of the BOLD response in left temporal cortex at the parietal-temporal-occipital (PTO) junction. On this basis they argued for a double dissociation between the neural circuits responsible for mental rotation and perspective taking. In a similar vein, Wraga et al. (2005) found low-level premotor activation consistent with preparatory hand movements during mental rotation alone, while perspective taking was uniquely associated with responses in the supplementary motor area and left middle occipital gyrus at the junction of the fusiform gyrus. Comparisons with control tasks revealed parietal (BA 7) activations associated with both types of transformation, but while these were bilateral for object rotations, they were restricted to right parietal for viewer rotations. Even so, while direct comparisons such as these have found distinct neural substrates associated with each task (as well as 
brain regions common to both), the unique regions identified are somewhat inconsistent across studies, especially for perspective taking in which there has sometimes been no detectable increase in activation above control (e.g., Zacks et al., 2002).

In light of the behavioral data, a question that arises is whether brain activations associated with mental rotation are specifically responsive to angle of rotation. In an early fMRI study of mental rotation, Carpenter et al. (1999) found linear trends such that regions in the left and right parietal cortex were increasingly activated with greater degrees of rotation, both in terms of number of voxels within a large region of interest and average amplitude of activation across voxels in the region. Interestingly, no study to our knowledge has investigated this question further with the more sophisticated neuroimaging methods now available, including event-related fMRI and whole brain voxel-wise analysis, which enables more precise localization. Recent studies have parametrically varied the number of mental rotation trials in a block of trials and found corresponding increases in activation in right posterior parietal cortex, centered on the intraparietal sulcus (Harris et al., 2000; Podzebenko et al., 2002). However, these studies did not examine the effects of magnitude or angle of rotation, and no neuroimaging studies to date have directly compared the effects of rotation magnitude in mental rotation and perspective taking.

The aim of the present study was to go beyond previous neuroimaging research in this field by directly comparing the relationship between magnitude of rotation and the hemodynamic response during both types of transformation in an event-related design. We used novel tasks in which participants viewed a rendering of a circular table with an object on top and imagined either that the table rotated or that they moved around the 
table to view it from a different location. Their task was to decide whether the object would be on their left or right after the spatial transformation. Note that whereas mental rotation has traditionally been studied using relatively simple rotations of objects about a single axis (e.g., Shepard \& Metzler, 1971), research on perspective taking has included tasks that involve both translations through space and rotations, sometimes about multiple axes (e.g., Burgess, Spiers, \& Paleologou, 2004; Shelton \& McNamara, 2004; Wang \& Simons, 1999). The tasks studied here involve imagined rotation and translation of either the self or object, but for simplicity we will refer to them as mental rotation and perspective taking, respectively.

An informal task analysis suggests that the following processes are involved in our tasks: (1) viewing the stimulus, (2) encoding the spatial location of the object, (3) executing the spatial transformation, (4) judging whether the object is on the right or left, (5) responding. As described above, an important property of this task is that the stimulus and mode of responding are identical for mental rotation and perspective taking, thus only the spatial transformation differs in the two versions of the task (cf. Wraga et al., 2005; Zacks et al., 2003).

To examine the neural activity involved in both tasks, including their common processes, we first compared all trials to rest. To isolate activations associated with just the spatial transformation, we next compared rotation trials to no-rotation trials, which involve all the common processes but no spatial transformation. To isolate which regions were responsive to angle of rotation for both mental rotation and perspective taking, we identified areas that exhibited a significant linear relationship between rotation magnitude and the hemodynamic response. Based on behavioral data and previous neuroimaging 
research, we predicted that mental rotation would be associated with a positive correlation between angle of rotation and neural response in right posterior parietal cortex. Given the inconsistent findings from behavioral research and the paucity of previous neuroimaging data on perspective taking, we did not make a priori predictions regarding neural response to angle of rotation for perspective taking.

If the neural mechanisms underlying self and object transformations are dissociated, as suggested by behavioral and psychometric data, there are at least two ways in which this may be evident. One possibility is that mental rotation and perspective taking will differentially activate separate regions (cf. Wraga et al., 2005; Zacks et al., 2003), which might further differ in terms of whether they exhibit parametric modulation of activation by rotation magnitude. An alternative possibility is that activation in the same regions might be differentially affected by angle of rotation for the two spatial transformations, as suggested by their different response time profiles.

Materials and Methods

Participants

Twenty right-handed Dartmouth College graduate students were paid \$20 for participating. Two participants' data were discarded due to excessive head movement and technical difficulties, respectively. Of the remaining 18, we analyzed only the 14 participants ( 7 males) with fewer than $20 \%$ errors in both conditions, to ensure that the participants analyzed were performing the tasks correctly.

Stimuli

Stimuli and responses were identical for the two conditions, and control trials were identical to rotation trials except that they did not require the execution of a spatial 
transformation, allowing us to isolate activations uniquely associated with each spatial transformation process. Task parameters were such that any attempt to solve a perspective taking trial with a reverse-direction mental rotation transformation, or vice versa, would result in incorrect responses at least some of the time. Thus, in terms of accuracy, it was not in participants' interest to substitute one type of transformation for the other during either condition.

Stimuli were created using 3D Studio Max software. The images represented a circular table with a ball on top, rendered as if viewed from a $45^{\circ}$ angle, so that participants could imagine they were looking at a tabletop in front of them. An arrow below the table indicated the direction and distance of the to-be-imagined transformation (the ball and arrow were always rendered on opposite sides of the table; see Figure 1a). Arrow lengths were $30^{\circ}, 60^{\circ}, 90^{\circ}, 120^{\circ}$, and $150^{\circ}$, plus $0^{\circ}$ control trials, requiring no rotation (see Figure 1b). For each rotation angle, the location of the ball on the table was systematically varied through all possible positions at $30^{\circ}$ intervals, other than those that precluded a left or right answer. Left and right versions of every rotation stimulus were rendered.

Procedure

A training period with self-paced instructions and 20 practice trials for each condition was conducted outside the scanner. Immediately before each condition 20 additional practice trials were presented in the scanner. To avoid additional brain activation due to task switching and the need for a condition cue on each trial, the mental rotation and perspective taking conditions were blocked. Presentation order of rotation angles was randomized within each block. Each condition block was presented in 3 
consecutive runs. Each run began with a fixation period of 24 seconds, followed by 60 event trials in fixed random order (different orders were used for each run). Fixation trials were pseudo-randomly inserted between event trials to enable efficient event-related analysis. Order of conditions and runs was counterbalanced.

In each event trial, the stimulus (e.g., Figure 1a) was presented for $3500 \mathrm{~ms}$, followed by a 500ms inter-stimulus interval. During the mental rotation blocks, participants were instructed to imagine that the table rotated the distance and direction indicated by the arrow, while they remained stationary. During the perspective taking blocks, participants were instructed to imagine that the table remained stationary, while they moved around it to the tip of the arrow and looked across the center of the table from there. In both conditions, the task was to decide whether the ball would be on their left or their right after the imagined transformation. Participants held one button in each hand. They pressed the left-hand button to indicate the ball would be on their left, and the righthand button to indicate the ball would be on their right. The maximum response time was $4000 \mathrm{~ms}$. Each of the rotation angles $\left(0^{\circ}, 30^{\circ}, 60^{\circ}, 90^{\circ}, 120^{\circ}, 150^{\circ}\right)$ appeared a total of 30 times in each condition, in a non-predictable quasi-random order. Imaging Procedures Imaging was conducted at Dartmouth College using a 1.5T GE SIGNA Echospeed MRI scanner (General Electric, Milwaukee, WI). Foam padding was used for head stabilization. Stimulus presentation was controlled by an Apple G3 laptop using PsyScope (Cohen, MacWhinney, Flatt, \& Provost, 1993). Stimuli were back-projected onto a screen at the head end of the scanner bore by an LCD projector; participants viewed the screen using a mirror mounted on the head coil. Participants made responses 
using two magnet-compatible fiberoptic button presses, one held in each hand, which communicated with the laptop via the PsyScope Button Box (Carnegie Mellon University, Pittsburgh, PA). Functional images were collected in 6 discrete runs (3 consecutive runs per condition, order of conditions counterbalanced across participants). Each run consisted of five blocks of task trials separated by four evenly spaced rest blocks comprising $24 \mathrm{~s}$ of fixation. 132 functional images were acquired during each run; the first 6 functional images from each run were discarded to allow steady-state magnetization to be approached. Functional images were acquired using a gradient spinecho, echo-planar sequence sensitive to the BOLD contrast $(\mathrm{TR}=4000 \mathrm{~ms}, \mathrm{TE}=35 \mathrm{~ms}$, flip angle $\left.=90^{\circ}\right) .25$ contiguous axial slices with a field of view of $24 \mathrm{~cm}$ were acquired to permit coverage of the whole brain (1mm skip), producing $3.75 \times 3.75 \times 5.5 \mathrm{~mm}$ voxels. T1-weighted structural images were acquired using a high-resolution 3D spoiled gradient recovery sequence ( 124 saggital slices, $\mathrm{TE}=6 \mathrm{~ms}$, flip angle $=25^{\circ}, 1 \times 1 \times 1.2$ $\mathrm{mm}$ voxels).

Functional MRI Data Analysis

Images were preprocessed using SPM2 (Wellcome Department of Imaging Neuroscience, University College London, UK). All functional images were registered to the first volume to correct for minor head movements and were then registered to the anatomical image. The anatomical image was then transformed to conform to MNI space and the parameters of this transformation were applied to the functional data (all images were resampled to $3 \mathrm{~mm}$ isotropic voxels). Finally, the functional data were smoothed using an $8 \mathrm{~mm}$ full-width at half-maximum Gaussian filter. 
Subsequent analysis was conducted using custom software written in MATLAB (The MathWorks, Natick, MA). The general linear model was used to analyze the fMRI time-series (Friston et al., 1995). Following Ollinger, Shulman, \& Corbetta (2001), each stimulus onset and post stimulus time point (up to a specified limit, in this case $20 \mathrm{sec}$.) was modeled by a separate parameter. There were five post-stimulus time bins covering a total window length of 20 seconds. This approach is very similar to selective averaging (Dale \& Buckner, 1997) in that it can be thought of as selective averaging without counterbalancing of trial orders. This model is also known as a finite impulse response model (Henson, Rugg, \& Friston, 2001). The benefit of this model is that it makes minimal assumptions about the shape of the hemodynamic response, thus accommodating variations in the timing of the response that have been observed across brain regions (e.g., Schacter, Buckner, W, Dale, \& Rosen, 1997) and avoiding the amplitude bias that these variations can introduce (Calhoun, Stevens, Pearlson, \& Kiehl, 2004).

Using this approach, four trial types were modeled: mental rotation, mental rotation control $\left(0^{\circ}\right)$, perspective taking, and perspective taking control $\left(0^{\circ}\right)$. Additionally, the magnitude of rotation required for each trial was modeled separately for mental rotation and perspective taking. This was done by replacing the "box-car" components of the design matrix with continuously varying predictor variables. In producing these predictor variables, the degrees of rotation were rescaled and centered at 0 to enable reasonably efficient estimation. In addition to the parameters already discussed, 6 parameters modeled linear drift within each session and 6 parameters modeled the session-specific means. 
Activation levels for each trial type were estimated by summing the estimated hemodynamic response across the $2^{\text {nd }}, 3^{\text {rd }}$, and $4^{\text {th }}$ time points. Exclusion of the earliest and latest components of the estimated hemodynamic response was done to accommodate undershoots in the response that are sometimes observed (we thank F.G. Ashby for suggesting this approach). An analogous approach was used to estimate the effect of degrees of rotation. Group maps of the $t$ statistic were computed by testing the mean activation level for a trial type, average activation across trial types, or differences in activation levels across trial types against zero. All contrasts were bi-directional, i.e., we tested for both positive and negative linear trends. Clusters in which 10 or more contiguous voxels survived a threshold of $p<.001$ (two-tailed; uncorrected for multiple comparisons) were declared statistically significant. We chose to use a threshold that would reveal any regions that might be sensitive to the parametric modulations of mental rotation or perspective taking, while still maintaining a statistical threshold and voxel extent that would survive a corrected probability criterion for clusters (Forman et al., 1995; Xiong, Gao, Lancaster, \& Fox, 1995). Plotted time courses represent the averages of parameter estimates across voxels within a cluster.

Results

\section{Behavioral Data}

After exclusion of high-error participants, mean errors constituted less than $7 \%$ of mental rotation trials and less than $5 \%$ of perspective taking trials; therefore RTs were used as the dependent measure. To minimize the effects of outliers, response time data were based on each subject's median RT for each angle. 
As Figure 1c shows, RTs for mental rotation increased linearly with magnitude of rotation, consistent with well-established linear RT patterns for mental rotation of objects (Shepard \& Metzler, 1971). By contrast, in perspective taking the function was nonmonotonic, which is consistent with previously documented RT patterns from imagined shifts of perspective (Creem et al., 2001; Wraga et al., 2000).

A $2 \times 5$ (condition by angle) repeated measures ANOVA revealed a main effect of angle on $\mathrm{RT}, F(4,10)=7.09, p=.006$, partial $\eta^{2}=.74$, and a significant interaction of condition by angle, $F(4,52)=4.53, p=.003$, partial $\eta^{2}=.26$. Separate within-subjects contrasts showed a significant linear trend for mental rotation, $F(1,13)=9.79, p=.008$, partial $\eta^{2}=.43$, but not for perspective taking, $F(1,13)=.87, p=.37$.

Neuroimaging data

We began with a whole-brain analysis to examine the areas activated in both tasks (relative to fixation). Mental rotation and perspective taking activated an extensive common area of cortex, most notably bilateral visual cortex, inferior temporal cortex, and superior parietal cortex (see Figure 2). This activation presumably reflects common processes such as viewing the stimulus, encoding the locations of ball and arrow, and making a response, as well as the transformation itself. We then compared rotation trials collapsed across all angles with control (no-rotation or $0^{\circ}$ ) trials to identify areas uniquely associated with the transformation process. For mental rotation this contrast implicated bilateral superior parietal cortex $(x=-42, y=-59, z=53$ BA $7 ; x=50, y=-36, z=43$ BA 40 ) and right middle frontal gyrus $(x=30, y=6, z=52$; BA 6), a premotor area associated with mental rotation of objects (Wraga et al., 2005). For perspective taking, no regions survived threshold for this contrast. 
We then identified which areas exhibited a significant linear relationship between rotation magnitude and the hemodynamic response. For mental rotation, a subset of regions near those previously implicated showed a positive linear relationship between degrees of rotation and the hemodynamic response: bilateral superior parietal cortex (BA 40/7) and a small cluster in right adjacent somatosensory cortex (BA 2; see Figure 3 and Table 1 upper section). For perspective taking, no areas survived the threshold. A direct comparison of the estimated linear effects showed that the linear trends for mental rotation and perspective taking differed significantly in a large region of right parietal cortex surrounding the intraparietal sulcus (BA 40/7), overlapping with areas implicated in the previous contrast, and in two clusters within right middle frontal gyrus (see Figure 4 and Table 1 lower section).

\section{Right Superior Parietal Cortex}

We further inspected the response in right superior parietal (BA 40), a large cluster surrounding the intraparietal sulcus that was implicated in the previous two contrasts. The estimated hemodynamic responses as a function of degrees of rotation, as implied by the model fit, are shown in Figure 4. This fit is based on an average of parameter estimates of all voxels in the cluster; fits for several individual voxels within the cluster were plotted to ensure the average was representative of the cluster. For mental rotation there was a positive correlation between degrees of rotation and the regional response, which is consistent with the previous contrast. However, for perspective taking there was a negative correlation between degrees of rotation and the regional response. 
To further inspect this finding, the data were collapsed across voxels in this cluster individually for each subject. Then a new model was estimated that treated each level of rotation magnitude as a separate trial type (producing 12 trial types in total; $d f=$ 683). Finally, the estimated peak response ( $2^{\text {nd }}$ post-stimulus time point) for each subject and for each trial type other than control trials $\left(0^{\circ}\right)$ was submitted to a repeated-measures ANOVA with a threshold of $p<.05$. This ANOVA indicated a condition by angle interaction with a significant linear component, $F(1,13)=31.64, p<.001$, partial $\eta^{2}=$ .71 , indicating that the linear trend differed by condition (see Figure 4). To inspect this interaction, ANOVAs were conducted separately for the two tasks. These analyses showed a significant positive linear trend for mental rotation, $F(1,13)=9.21, p=.01$, partial $\eta^{2}=.42$, and a significant negative linear trend for perspective taking, $F(1,13)=$ $36.66, p<.001$, partial $\eta^{2}=.74$ (see Figures 4 and 5). No higher order polynomial component approached significance.

\section{Discussion}

In this study, we used novel tasks to study the processes involved in spatial transformations of self and objects. Response times were linearly related to magnitude of rotation in the mental rotation task but not in the perspective-taking task. Both tasks activated an extensive common area of cortex, including bilateral visual cortex, inferior temporal cortex, and superior parietal cortex. Such activation presumably reflects not only the spatial transformation, but also common processes such as viewing the stimulus and encoding the locations of ball and arrow.

When no-rotation trials were subtracted from rotation trials, we found activations associated with mental rotation in bilateral superior parietal cortex, suggesting that these 
areas are uniquely associated with the mental rotation transformation process. The localization of mental rotation processes to these areas is consistent with previous research (Carpenter et al., 1999; Cohen et al., 1996; Jordan, Heinze, Lutz, Kanowski, \& Janke, 2001; Wraga et al., 2005; Zacks et al., 2003), indicating that the same regions are activated during mental rotation in our novel paradigm as in more classic mental rotation tasks, such as the Shepard \& Metzler paradigm. Furthermore, we found a positive linear relationship between degrees of rotation and the hemodynamic response in these regions, a finding that supports the idea that these areas were responsible for the transformation process itself, rather than other aspects of the task. The positive linear trend supports the characterization of mental rotation as a continuous analog transformation. It is not readily interpreted simply as a time on task effect. If the positive linear trend merely reflected time on task, all regions activated by this task should demonstrate this pattern, yet a variety of areas implicated in mental rotation did not (cf. Figure 2 and Table 1). The linear trend is seen in only a subset of the regions activated in the mental rotation task, isolating these as areas that are implicated in the spatial transformation.

A closer inspection of one of the regions showing a linear trend for mental rotation, right superior parietal (BA 40) ${ }^{1}$, indicated that the direction of the linear trend differed according to the type of spatial transformation. In this region both tasks exhibited a linear relationship between degrees of rotation and the hemodynamic response, but whereas mental rotation was associated with a positive linear trend, quite surprisingly, the direction of the linear relationship for perspective taking was negative.

This raises the question of the role of this region in mental rotation and perspective taking. Our experimental task involved making an egocentric judgment, to 
decide whether the ball would be to the left or the right of the body midline after the spatial transformation. As part of the dorsal stream, a key function of parietal cortex is to code the locations of objects in relation to the body, allowing us to act on the world (Milner \& Goodale, 1995). As such, the parietal lobes contain multiple representations of space in body-based or egocentric frames of reference. Regions centered on the intraparietal sulcus are known to locate targets in relation to eye-centered, head-centered, body-centered, and limb-centered coordinates, and in posterior parietal cortex this information is transformed into a common egocentric spatial reference frame (for reviews, see Bremmer, 2005; Cohen \& Anderson, 2002; Colby, 1998; Grefkes \& Fink, 2005). The region in which we found opposing linear trends for mental rotation and perspective taking overlaps with the intraparietal sulcus (see Figure 4).

During mental rotation, it seems plausible that this region might be performing an updating function, to recalibrate the imagined new position of the ball with respect to the viewer, allowing them to decide whether it will be on their left or right side after the table has rotated. The greater the imagined movement of the ball from its starting position, the greater the required updating of its position in relation to body-centered coordinates, and hence the positive linear trend we observed in this region.

During perspective taking, on the other hand, the viewer attempts to imagine the scene from a position other than his or her actual viewpoint. There are two components to this process. In order to generate a projected perspective somewhere else in space, they must not only assume the imagined perspective, but they must also disregard their actual perspective. Several lines of evidence suggest that a viewer's actual perspective can interfere with their ability to generate and maintain a projected perspective (May, 2004; 
Waller, Montello, Richardson, \& Hegarty, 2002). In perspective-shifting tasks, errors are egocentric not random (Huttenlocher \& Presson, 1973, 1979); performance improves if the viewer is disoriented such that their knowledge of their actual body position is disrupted (Waller et al., 2002); and performance is worse if the mode of responding (e.g., pointing) anchors the viewer back to their physical body orientation (Avraamides, Klatzky, Loomis, \& Gollege, 2004; Wraga, 2003). Thus, it is only by ignoring or suppressing information about one's actual position in space that one can successfully project oneself into a different, imagined position in space. If right superior parietal cortex codes self-to-object spatial relations, it should be involved in the initial encoding phase of the task, but its role might be expected to decrease the further one imagines shifting away from one's actual position. One highly speculative account is that the linear decrease in parietal activation observed for greater imagined perspective change reflects the need to disregard one's physical body orientation, and its true relation to objects in the environment, during this task.

Even if we accept this speculative account, suppressing or disregarding one's current body position is only part of the perspective-taking process; one also has to imagine a shift to a new location or orientation, and this transformation should give rise to associated activation. Yet the activation associated with perspective taking, when collapsed across all rotation angles, was not significantly greater than that observed in the control (no rotation) trials. This null result might seem at odds with the significant linear trend observed in this condition, but in fact it is quite possible to have a significant linear trend and yet have overall (averaged) activation that does not exceed control trial levels, if the linear trend causes some responses to fall below control levels and some above, as 
occurred here. In a post-hoc analysis we directly compared perspective taking for angles greater and less than 90 degrees $^{2}$, which were found to be dissociated in a previous psychometric study (Kozhevnikov \& Hegarty, 2001), but again no areas survived threshold. Our findings are not altogether inconsistent with previous studies, some of which have found no regions associated with perspective taking that responded above control, even when using a more lenient statistical threshold than was employed here (e.g., Zacks et al., 2002).

There are several factors that might have contributed to the null result in the present study. First, consistent with previous studies (e.g., Wraga et al., 2000) there was a trend for perspective taking to take less time than mental rotation, making it more difficult to detect unique activation associated with this task with fMRI (consistent with the RT trends, in post-task self-reports participants rated perspective taking as significantly easier than mental rotation). Second, both previous research (Kozhevnikov \& Hegarty, 2001) and the self-reports of participants in this study suggest that the strategies employed in the perspective taking condition were much more varied than in the mental rotation condition. In mental rotation, all participants reported an analog transformation of the stimulus through essentially the same path or trajectory; that is, they imagined the ball rotating about the center of the table in a circular motion corresponding to the direction and distance indicated by the arrow (one participant reported imagining the arrow rotating back to the ball, but this still involved the same movement trajectory). By contrast, in the perspective taking condition, six participants reported that they imagined themselves moving or walking around the table in a circular path to the tip of the arrow, five participants reported that they imagined themselves instantly repositioned 
at the arrow tip, without traveling around the table to get there, and three participants described a mixture of these strategies. A post-hoc comparison of the behavioral data revealed RT patterns that were broadly consistent with these two reported paths. Given that perspective taking appears to be amenable to different strategies, or different imagined movement trajectories, common regions of activation might be difficult to find when averaging over participants. Thus, it is also possible that our failure to find significant activation associated with perspective shifting is a Type II error due to low power in the present study.

In future studies it will be important to examine more challenging perspective taking tasks and to experimentally dissociate areas involved in assuming a new perspective from those involved in suppressing one's current perspective. The results of this study also indicate that future research should specifically investigate the activations associated with different perspective taking strategies either by correlating the hemodynamic response with post-task reports or by inducing different strategies through task instructions, as has been done in studies of mental rotation (e.g., Kosslyn, Thompson, Wraga, \& Alpert, 2001). In these studies it will also be important to contrast the effects of angle of rotation on spatial transformations of objects and the self using tasks that do not require an egocentric (left/right) body-relative judgment, such as tasks in which the viewer chooses which of several pictures shows the appearance of an array after the imagined spatial transformation (cf. Huttenlocher \& Presson, 1973, 1979).

In conclusion, the present data are consistent with previous research implicating the superior parietal lobe in mental rotation and with the characterization of mental rotation as a continuous analog transformation. They also demonstrate that the right 
superior parietal cortex is specifically responsive to the magnitude of rotation, suggesting that this area is involved in the continuous updating of self-to-object spatial relations. This region also makes a contribution to perspective taking, but our data offer the intriguing possibility that the nature of this contribution is qualitatively different. These findings offer a window into the egocentric representation of space in human parietal cortex, and the role of this region in spatial transformations. 


\section{References}

Avraamides, M. N., Klatzky, R. L., Loomis, J. M., \& Gollege, R. G. (2004). Use of cognitive versus perceptual heading during imagined locomotion depends on response mode. Psychological Science, 15, 403-408.

Bremmer, F. (2005). Navigation in space - the role of the macaque ventral intraparietal area. Journal of Physiology, 566(1), 29-35.

Burgess, N., Spiers, H. J., \& Paleologou, E. (2004). Orientational manoeuvres in the dark: dissociating allocentric and egocentric influences on spatial memory. Cognition, 94, 149-166.

Calhoun, V. D., Stevens, M. C., Pearlson, G. D., \& Kiehl, K. A. (2004). fMRI analysis with the general linear model: removal of latency-induced amplitude bias by incorporation of hemodynamic derivative terms. NeuroImage, 22, 252-257.

Carpenter, P. A., Just, M. A., Keller, T. A., \& Eddy, W. (1999). Graded functional activation in the visuospatial system with amount of task demand. Journal of Cognitive Neuroscience, 11(1), 9-24.

Carroll, J. (1993). Human cognitive abilities: A survey of factor-analytic studies. New York: Cambridge University Press.

Cohen, J. D., MacWhinney, B., Flatt, M., \& Provost, J. (1993). PsyScope: a new graphic interactive environment for designing psychology experiments. Behavioral Research Methods, Instruments, and Computers, 25, 257-271.

Cohen, M. S., Kosslyn, S. M., Breiter, H. C., DiGirolamo, G. J., Thompson, W. L., Anderson, A. K., et al. (1996). Changes in cortical activity during mental rotation. A mapping study using functional MRI. Brain, 119, 89-100.

Cohen, Y. E., \& Anderson, R. A. (2002). A common reference frame for movement plans in the posterior parietal cortex. Nature Reviews Neuroscience, 3, 553-562.

Colby, C. L. (1998). Action-oriented spatial reference frames in cortex. Neuron, 20, 1524.

Creem, S. H., Downs, T. H., Wraga, M., Harrington, G. S., Proffitt, D. R., \& Downs, J. H. (2001). An fMRI study of imagined self-rotation. Cognitive, Affective, \& Behavioral Neuroscience, 1(3), 239-249.

Dale, A. M., \& Buckner, R. L. (1997). Selective averaging of rapidly presented individual trials using fMRI. Human Brian Mapping, 5, 329-340.

Easton, R. D., \& Sholl, M. J. (1995). Object-array structure, frames of reference, and retrieval of spatial knowledge. Journal of Experimental Psychology: Learning, Memory, and Cognition, 21(2), 483-500.

Forman, S., Cohen, J., Fitzgerald, M., Eddy, W., Mintun, M., \& Noll, D. (1995). Improved assessment of significant activation in functional magnetic resonance imaging (fMRI): Use of a cluster-size threshold. Magnetic Resonance in Medicine, 33(5), 636-647.

Friston, K. J., Holmes, A. P., Worsley, K. J., Poline, J. P., Frith, C. D., \& Frackowiak, R. S. J. (1995). Statistical parametric maps in functional imaging: a general linear approach. Human Brain Mapping, 2, 189-210.

Grefkes, C., \& Fink, G. R. (2005). The functional organization of the intraparietal sulcus in humans and monkeys. Journal of Anatomy, 207, 3-17. 
Harris, I. M., Egan, G. F., Sonkkila, C., Tochon-Danguy, H. J., Paxinos, G., \& Watson, J. D. G. (2000). Selective right parietal lobe activation during mental rotation. Brain, 123, 65-73.

Hegarty, M., \& Waller, D. (2004). A dissociation between mental rotation and perspective-taking spatial abilities. Intelligence, 32, 175-191.

Henson, R. N. A., Rugg, M. D., \& Friston, K. J. (2001). The choice of basis functions in event-related fMRI. NeuroImage, 13, 149.

Huttenlocher, J., \& Presson, C. C. (1973). Mental rotation and the perspective problem. Cognitive Psychology, 4, 277-299.

Huttenlocher, J., \& Presson, C. C. (1979). The coding and transformation of spatial information. Cognitive Psychology, 11, 375-394.

Jordan, K., Heinze, H. J., Lutz, K., Kanowski, M., \& Janke, L. (2001). Cortical activations during the mental rotation of different visual objects. NeuroImage, 13, 143-152.

Kosslyn, S. M., Thompson, W. L., Wraga, M., \& Alpert, N. M. (2001). Imagining rotation by endogenous versus exogenous forces: Distinct neural mechanisms. Neuroreport, 12, 2519-2525.

Kozhevnikov, M., \& Hegarty, M. (2001). A dissociation between object manipulation spatial ability and spatial orientation ability. Memory \& Cognition, 29(5), 745756.

May, M. (2004). Imaginal perspective switches in remembered environments: Transformation versus interference accounts. Cognitive Psychology, 48, 163-206.

Milner, D. A., \& Goodale, M. A. (1995). The Visual Brain in Action. New York: Oxford University Press.

Ollinger, J. M., Shulman, G. L., \& Corbetta, M. (2001). Separating processes within a trial in event-related functional MRI. NeuroImage, 13, 210-217.

Podzebenko, K., Egan, G. F., \& Watson, J. D. G. (2002). Widespread dorsal stream activation during a parametric mental rotation task, revealed with functional magnetic resonance imaging. Neurolmage, 15, 547-558.

Reiser, J. J. (1989). Access to knowledge of spatial structure at novel points of observation. Journal of Experimental Psychology: Learning, Memory, and Cognition, 15(6), 1157-1165.

Richter, W., Somorjai, R., Summers, R., Jarmasz, M., Menon, R. S., Gati, J. S., et al. (2000). Motor area activity during mental rotation studied by time-resolved single-trial fMRI. Journal of Cognitive Neuroscience, 12(2), 310-320.

Schacter, D. L., Buckner, R. L., W, K., Dale, A. M., \& Rosen, B. R. (1997). Late onset of anterior prefrontal activity during true and false recognition: An event-related fMRI study. NeuroImage, 6(4), 259-269.

Shelton, A. L., \& McNamara, T. P. (2004). Spatial memory and perspective taking. Memory \& Cognition, 32(3), 416-426.

Shepard, R. N., \& Metzler, J. (1971). Mental rotation of three-dimensional objects. Science, 171, 701-703.

Waller, D., Montello, D. R., Richardson, A. E., \& Hegarty, M. (2002). Orientation specificity and spatial updating of memories for layouts. Journal of Experimental Psychology: Learning, Memory, and Cognition, 28(6), 1051-1063. 
Wang, R. F., \& Simons, D. J. (1999). Active and passive scene recognition across views. Cognition, 70, 191-210.

Wraga, M. (2003). Thinking outside the body: An advantage for spatial updating during imagined versus physical self rotation. Journal of Experimental Psychology: Learning, Memory and Cognition, 29(5), 993-1005.

Wraga, M., Creem, S. H., \& Proffitt, D. R. (2000). Updating displays after imagined object and viewer rotations. Journal of Experimental Psychology: Learning, Memory, and Cognition, 26, 151-168.

Wraga, M., Shepard, J. M., Church, J. A., Inati, S., \& Kosslyn, S. M. (2005). Imagined rotations of self versus objects: an fMRI study. Neuropsychologia, 43(9), 13511361.

Xiong, J., Gao, J.-H., Lancaster, J. L., \& Fox, P. T. (1995). Clustered pixels analysis for functional MRI activation studies of the human brain. Human Brain Mapping, 3(4), 287-301.

Zacks, J. M., \& Michelon, P. (2005). Transformations of visuospatial images. Behavioral and Cognitive neuroscience Reviews, 4(2), 96-118.

Zacks, J. M., Ollinger, J. M., Sheridan, M. A., \& Tversky, B. (2002). A parametric study of mental spatial transformations of bodies. Neurolmage, 16, 857-872.

Zacks, J. M., Rypma, B., Gabrieli, J., Tversky, B., \& Glover, G. (1999). Imagined transformations of bodies: An fMRI study. Neuropsychologia, 37, 1029-1040.

Zacks, J. M., Vettel, J. M., \& Michelon, P. (2003). Imagined viewer and object rotations dissociated with event-related fMRI. Journal of Cognitive Neuroscience, 15(7), 1002-1018. 


\section{Footnotes}

${ }^{1}$ We focused on this region because it was larger than the other regions showing a linear trend for mental rotation, but an examination of the frontal regions exhibiting this linear relationship revealed the same general pattern.

${ }^{2}$ Imagined self-rotations of more than 90 degrees reverse egocentric left and right, and thus might involve a qualitatively different process from imagined self-rotations of less than 90 degrees. 


\section{Author Note}

Madeleine Keehner, School of Psychology, Curtin University of Technology;

Scott A. Guerin, Michael B. Miller, Mary Hegarty, Department of Psychology, University of California, Santa Barbara; David J. Turk, School of Psychology, University of Aberdeen.

This research was supported by the Center for Brain Imaging, University of California, Santa Barbara, CA. We thank Thomas Wolbers for his very helpful comments and suggestions on earlier drafts, and two anonymous reviewers, whose feedback helped to clarify ambiguities and improve our discussion of the findings.

Correspondence concerning this article should be addressed to: Madeleine Keehner, School of Psychology, Curtin University of Technology, GPO Box U 1987, Perth, WA 6845, Australia. Email: m.keehner@,curtin.edu.au 
Table 1: Regions Exhibiting a Positive Linear Relationship between Degrees of Rotation and the Hemodynamic Response

\begin{tabular}{lrrrlccc}
\hline Contrast & \multicolumn{3}{c}{ Maxima } & \multicolumn{1}{c}{ Region } & BA & $\boldsymbol{t}$ & Extent \\
& $\boldsymbol{x}$ & $\boldsymbol{y}$ & $\boldsymbol{z}$ & & & \\
\hline MR $>\mathbf{0}$ & 42 & -44 & 57 & R. Inferior Parietal Lobule & 40 & 6.33 & 42 \\
& 30 & -59 & 44 & R. Superior Parietal Lobule & 7 & 5.87 & 27 \\
& -42 & -45 & 38 & L. Inferior Parietal Lobule & 40 & 5.82 & 14 \\
& 56 & -21 & 45 & R. Postcentral Gyrus & 2 & 5.89 & 13 \\
& -48 & -33 & 49 & L. Postcentral Gyrus & 40 & 5.96 & 11 \\
& -30 & -58 & 61 & L. Superior Parietal Lobule & 7 & 5.64 & 11 \\
\hline MR $>$ PT & 53 & -41 & 52 & R. Inferior Parietal Lobule & 40 & 6.57 & 101 \\
& 48 & 39 & 20 & R. Middle Frontal Gyrus & 46 & 5.60 & 20 \\
& 27 & -73 & 56 & R. Precuneus & 7 & 4.72 & 14 \\
& 50 & 13 & 32 & R. Middle Frontal Gyrus & 9 & 6.58 & 13 \\
\hline
\end{tabular}

Note. $\mathrm{MR}=$ mental rotation; $\mathrm{PT}=$ perspective taking; BA $=$ Brodmann's area based on stereotaxic coordinates. 
Figure captions

Figure 1. Sample stimuli and behavioral data. (a) Rotation stimulus; (b) Control stimulus;

(c) Mean RTs at each rotation angle (+/-1 s.e.m.).

Figure 2. T-maps showing activation in each task (versus fixation baseline) overlaid on a $3 \mathrm{D}$ rendering of the mean anatomical image. Plots show the estimated hemodynamic response in a suprathreshold cluster within superior parietal cortex, indicated by the red arrow (+/- 1 s.e.m.).

Figure 3. T-map showing regions exhibiting a linear relationship between degrees of rotation and the hemodynamic response in the mental rotation task overlayed on a mean anatomical image. Plots show the estimated hemodynamic response as a function of degrees of rotation in four clusters of interest. The plots were obtained by averaging parameter estimates across all voxels within the cluster and deriving predicted response magnitudes for each angle from the model.

Figure 4. T-map showing regions exhibiting significantly different linear relationships between degrees of rotation and the hemodynamic response in the mental rotation task and in the perspective taking task, overlaid on a mean anatomical image. The first two plots show the estimated hemodynamic response as a function of degrees of rotation. The third plot shows the same data but restricts attention to the peak; this makes the size of the effect more readily apparent. The plots were obtained by averaging parameter 
estimates across all voxels within the cluster and deriving predicted activation magnitudes for each angle from the model.

Figure 5. Analysis of the response within a large region of right parietal cortex showing a positive relationship between angles of rotation and observed response for the mental rotation task but a negative relationship for the perspective taking task. Plots show estimates of the peak response ( $2^{\text {nd }}$ peristimulus time point) as a function of degrees of rotation for both tasks, collapsing across all voxels within the cluster and treating each angle as a separate trial type. The solid line indicates a simple linear regression; the error bars indicate $+/-1$ s.e.m. 


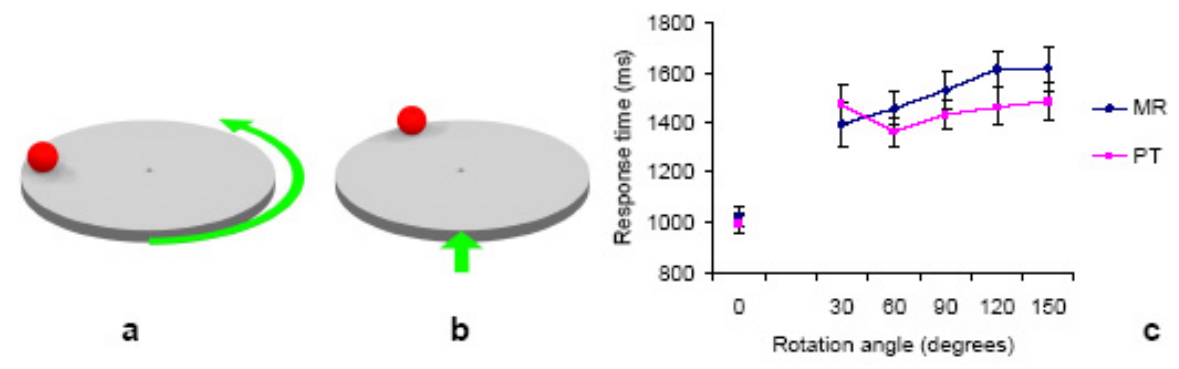





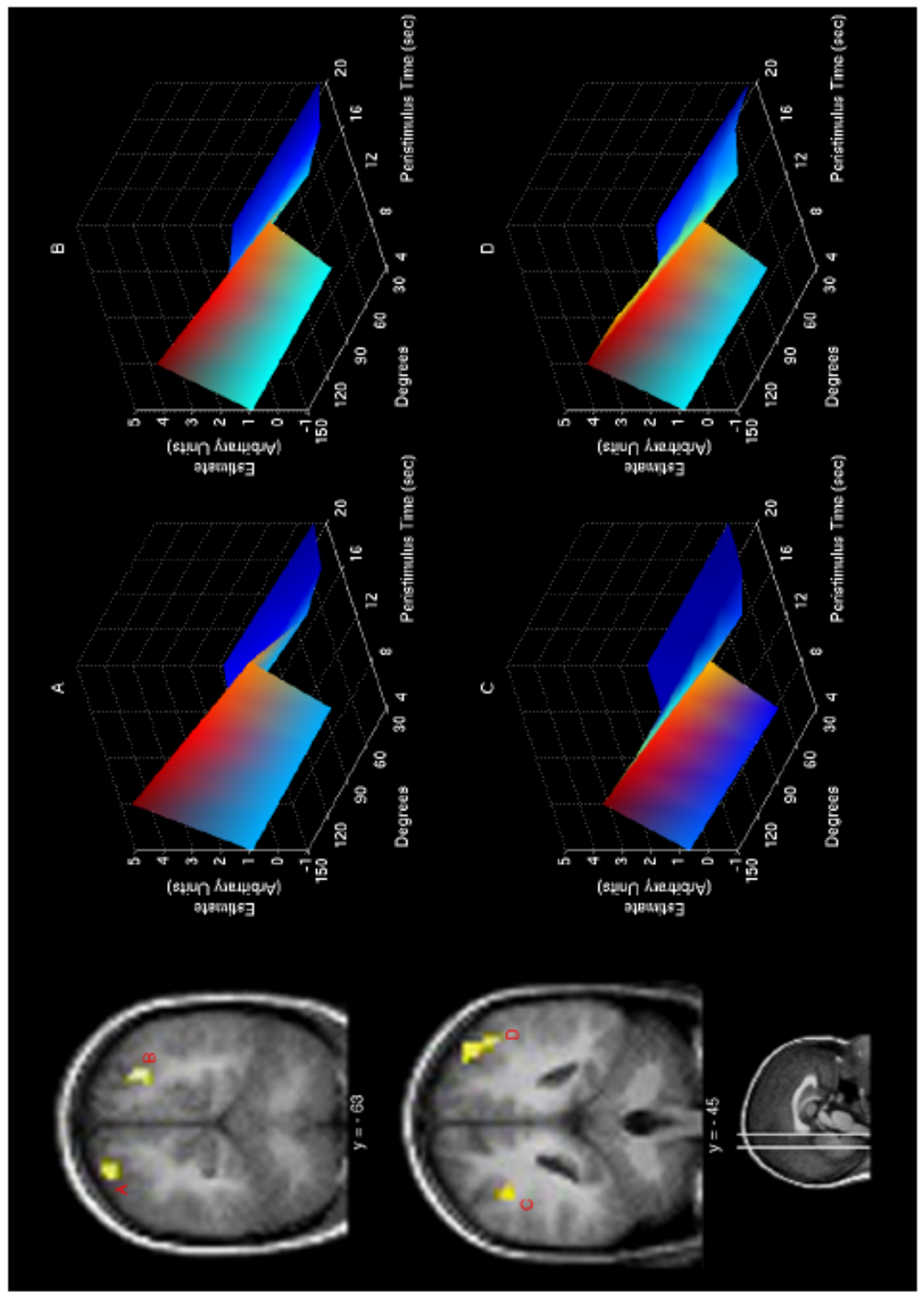




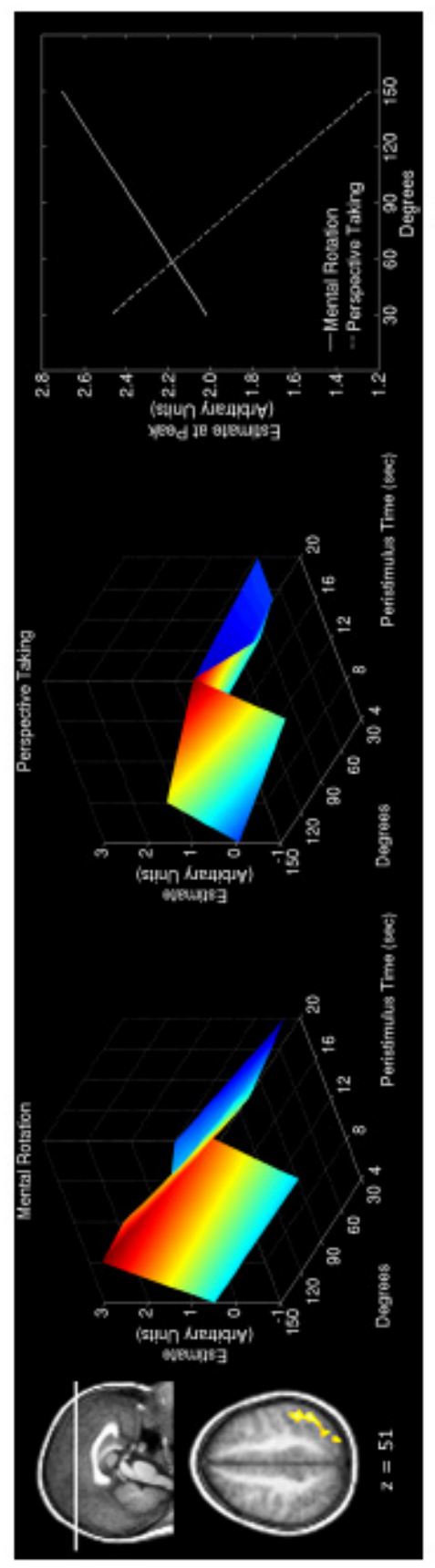

กี 

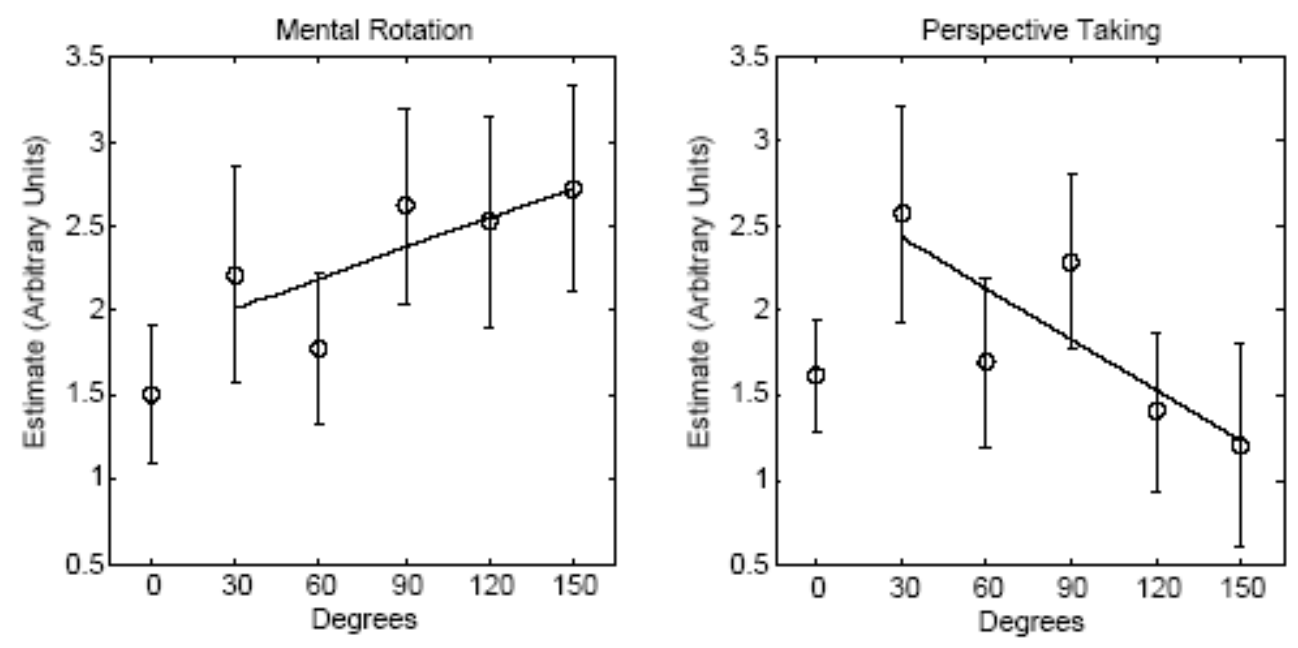